\title{
The Improvement of The Total Flow-Time Problem under The Non-Idle Machine Constraint of Two Machine Open-Shop
}

\author{
Liu Lin \\ Zhengzhou Institute Of Aeronautical Industry Management \\ Zhengzhou Henan,450015,China
}

\begin{abstract}
The total flow-time problem of two machine open-shop is NP-hard in strong sense. For the above problem without constrains, an explicit solution is constructed in this paper from the optimal schedule under the non-idle machine constraint, its total flow-time is reductive. The explicit solution under non-idle machine constraints must not be the explicit solution without constraints is proved under the consideration.
\end{abstract}

Keywords-Total flow-time, Open-shop, Efficient algorithm, Scheduling

\section{INTRODUCTION}

For $n$ job $J_{j}(j=1,2, \cdots, n)$ will be working in $m$ machine $M_{i}(i=1,2, \cdots, m)$, and all job are worked once on any machine, also working sequence is arbitrarily. It is called open-shop. Achugbue and Chin [2] have proved that $O 2 \| \sum c_{j}$ is NP-hard in strong sense. For the problem of the total flow-time problem of two machine open-shop that working time rely on the machine, is denoted by

$$
O 2\left|P_{i j}=P_{i}\right| \sum C_{j}
$$

For problem(1.1), M.Dror [3] give the optimal solution under $2 P_{2} \leq P_{1}$, and give a efficient algorithm to following problem

$$
O 2\left|P_{i j}=P_{i}, P_{2}<P_{1}<2 P_{2}\right| \sum C_{j}
$$

Siming Xiang and Guochun Tang [4]proved that M.Dror algorithm is wrong conclusion. They proved that the problem can transform a assignment problem, then

$$
O 2 \mid P_{i j}=P_{i}, P_{2}<P_{1}<2 P_{2}, \text { Non-Idle } \mid \sum C_{j}
$$

is polynomial solvable, Hungary algorithm i.e. Wenci $\mathrm{Yu}$ and Gang Ying[5]give the explicit solution for the problem. In this paper, an explicit solution of problem (2)is constructed and its total flow-time is more reductive than problem (3). It show that the proved that explicit solution of problem (2)is not explicit solution of problem (3).

Following are definitions and marks.

Definition 1 For schedule S, if completion time of job $\mathrm{j}$ in $M_{1}$ is smaller than in $M_{2}$, then we called job $\mathrm{j}$ is forward job, otherwise backward job.

Definition2 The cycling sub-schedule of problem(1.3),corresponding job subset is

\author{
Liu Weifeng \\ Zhengzhou Institute Of Aeronautical Industry Management \\ Zhengzhou Henan, 450015, China
}

$\{r, r+1, \cdots, r+l-1\}$, and $r$ is called the origination job, $l$ is called the length,processing sequencing as follows.

In $M_{1}$ : processing sequencing of jobs is $\{r, r+1, \cdots, r+l-1\}$,

In $M_{2}$ : processing sequencing of jobs is $\{r+1, \cdots, r+l-1, r\}$.

Definition3 The intersecting sub-schedule of problem(1.3), corresponding job subset is $\{r, r+1, r+2, r+3, r+4\}$, and $r$ is called the origination job, its length is 5,processing sequencing as follows.

In $M_{1}$ : processing sequencing of jobs is $\{r, r+1, r+2, r+3, r+4\}$.

In $M_{2}$ : processing sequencing of jobs is $\{r+1, r+3, r, r+4, r+2\}$.

Definition4 The parallel sub-schedule of problem(1.3) $\operatorname{Ir}(l)$,corresponding job subset is $\{r, r+1, \cdots, r+l-1\}$, and $r$ is called the origination job, $l$ is called the length, processing sequencing as follows.

In $M_{1}$ : processing sequencing of jobs is $\{r, r+1, \cdots, r+l-1\}$,

In $M_{2}$ : processing sequencing of jobs is $\{r, r+1, \cdots, r+l-1\}$.

The definitions and marks apply to problem(1.1)and problem 1.2).

\section{MAIN RESULTS}

For problem(1.3),namely $O 2 \mid P_{i j}=P_{i}, P_{2}<P_{1}<2 P_{2}$, Non-Idle $\mid \sum C_{j} \quad$,suppose $\mathrm{m}=\left\lceil\frac{P_{2}}{P_{1}-P_{2}}\right\rceil$, then $\frac{m+1}{m} P_{2} \leq P_{1} \leq \frac{m}{m-1} P_{2}$.

Theorem $1^{[5]}$ If the number of jobs $n$ satisfy $3 \leq n \leq m$, the optimal schedule $S_{*}$ of problem (1.3)is listed below.

(1)If $n \equiv 0(\bmod 3)$, then $S_{*}=T(3) T(3) \cdots T(3)$; 
(2)If $n \equiv 1(\bmod 3)$, then $S_{*}=T(4) T(3) \cdots T(3)$;

(3)If $n \equiv 2(\bmod 3)$, then $S_{*}=T(3) T(3) \cdots T(3) Q(5)$.

Theorem $2^{[5]}$ If the number of jobs $n$ satisfy $3 \leq n \leq m n>m$, the optimal schedule $S_{*}$ of problem (1.3)is listed below.

(1)If $m \equiv 0(\bmod 3)$, then

$S_{*}=T(3) T(3) \cdots T(3) I(n-m)$;

(2)If $m \equiv 1(\bmod 3)$, then

$S_{*}=T(4) T(3) \cdots T(3) I(n-m)$;

(3)If $m \equiv 2(\bmod 3)$, then

$S_{*}=T(3) T(3) \cdots T(3) I(n-m-1)$.

Lemma 1 If the number of jobs $n$ satisfy $3 \leq n \leq m$, the total flow-time of the optimal schedule $S_{*}$ of problem (1.3)is listed below.

$$
\begin{aligned}
\text { (1)If } n & =3 k \text {, then } \sum C_{j}=\frac{3}{2} k(k+1) P_{2}+k(3 k+2) P_{1} \\
\text { (2)If } n & =3 k+1 \text {, then } \\
\sum C_{j} & =\frac{1}{2} k(3 k+5) P_{2}+\left(3 k^{2}+4 k+2\right) P_{1} \\
\text { (3)If } n & =3 k+2, \text { then } \\
\sum C_{j} & =\left(\frac{3}{2} k^{2}+\frac{9}{2} k+2\right) P_{2}+\left(3 k^{2}+5 k+3\right) P_{1} .
\end{aligned}
$$

Proof (1) Let $I=\{$ Forward jobs subscript of the optimal schedule $\left.S_{*}\right\}$,

schedule $\left.S_{*}\right\}$,

$$
\bar{I}=\{\text { Backward jobs subscript of the optimal }
$$

Then $I=\{3 \mu+1 \mid \mu=0,1, \cdots, k-1\}$,

$\bar{I}=\{3 \mu+2,3 \mu+3 \mid \mu=0,1, \cdots, k-1\}$,

and $\sum_{j \in I} C_{j}=\sum_{\mu=0}^{k-1} 3(\mu+1) P_{2}=\frac{3}{2} k(k+1) P_{2}$,

$$
\sum_{j \in \bar{I}} C_{j}=\sum_{\mu=0}^{k-1}\left[(3 \mu+2) P_{1}+(3 \mu+2) P_{1}\right]=k(3 k+2) P_{1},
$$

Therefore

$\sum C_{j}=\sum_{j \in I} C_{j}+\sum_{j \in \bar{I}} C_{j}=\frac{3}{2} k(k+1) P_{2}+k(3 k+2) P_{1}$.

The proof of(2)and(3)is slightly.

We improved the optimal schedule in theorem 1 allowing in the machine idle period of time below.

First, we consider $n=3 k$.After completion of the processing job $3 l$, processing job $3 l+1$ in $M_{2}$ in first thing, after a period of time $t=(3 l+1) P_{2}-3 l P_{1}$, processing job $3 l+1,3 l+2, \cdots, 3 k$ in $M_{1}$ continuously, processing job $3 l+2,3 l+3, \cdots, 3 k$ in $M_{2}$ continuously. It is that turn

schedule $S_{*}$ to $S_{1}=T_{1}(3) T_{4}(3) \cdots T_{3 l-2}(3) I_{3 l+1}(3 k-3 l)$, among $S_{1}$ there are idle time $t=(3 l+1) P_{2}-3 l P_{1}$ in $M_{1}$ after completion of the processing job $3 l$.

Lemma 2 The total flow-time of the schedule

$$
S_{1}=T_{1}(3) T_{4}(3) \cdots T_{3 l-2}(3) I_{3 l+1}(3 k-3 l)
$$

$$
\begin{aligned}
\text { is } & \sum C_{j}=\frac{3}{2} l(l+1) P_{2}+l(3 l+2) P_{1} \\
& +(k-l)\left[\frac{1}{2}(9 l+9 k+3) P_{1}+9 l P_{2}-9 l P_{1}+3 P_{2}\right]
\end{aligned}
$$

Proof $\sum_{j \leq 3 l} C_{j}=\frac{3}{2} l(l+1) P_{2}+l(3 l+2) P_{1}$,

$$
\sum_{j>3 l} C_{j}=\sum_{i=1}^{3 k-3 l}\left[(3 l+i) P_{1}+3 l\left(P_{2}-P_{1}\right)+P_{2}\right]
$$

$=(k-l)\left[\frac{1}{2}(9 l+9 k+3) P_{1}+9 l P_{2}-9 l P_{1}+3 P_{2}\right]$

And $\sum C_{j}=\sum_{j \leq 3 l} C_{j}+\sum_{j>3 l} C_{j}$.

Theorem 3 Let $v=\frac{k}{5}+\frac{3 P_{2}-P_{1}}{15\left(P_{1}-P_{2}\right)}$, when $l$ satisfy $v<l<k$, the total flow-time of the schedule $S_{1}=T_{1}(3) T_{4}(3) \cdots T_{3 l-2}(3) I_{3 l+1}(3 k-3 l)$ is lesser than the schedule $S_{*}=T(3) T(3) \cdots T(3)$.

Proof When $v=\frac{k}{5}+\frac{3 P_{2}-P_{1}}{15\left(P_{1}-P_{2}\right)}<l<k$,

$$
\begin{aligned}
& \frac{1}{2}(9 l+9 k+3) P_{1}+9 l P_{2}-9 l P_{1}+3 P_{2}< \\
& \frac{3}{2}(k+l+1) P_{2}+(3 k+3 l+2) P_{1},
\end{aligned}
$$

Therefore the total flow-time of the schedule

$$
S_{1}=T_{1}(3) T_{4}(3) \cdots T_{3 l-2}(3) I_{3 l+1}(3 k-3 l)
$$

is lesser than the schedule $S_{*}=T(3) T(3) \cdots T(3)$ from lemma1 and lemma 2.

Second, we consider $n=3 k+1$. After completion of the processing job $3 l+4$, there is a period of idle time $t=(3 l+5) P_{2}-(3 l+4) P_{1}$ in $M_{1}$ and processing job $3 l+5,3 l+6, \cdots, 3 k+1$ in $M_{1}$ continuously and processing job $3 l+5,3 l+6, \cdots, 3 k+1$ in $M_{2}$ continuously. It is that turn schedule $S_{*}$ to $S_{2}=$ $T_{1}(4) T_{5}(3) \cdots T_{3 l+2}(3) I_{3 l+5}[3(k-l-1)]$, among $S_{2}$ there are idle time $t=(3 l+5) P_{2}-(3 l+4) P_{1}$ in $M_{1}$ after completion of the processing job $3 l+4$.

Lemma 3 The total flow-time of the schedule 
is

$$
\begin{aligned}
& S_{2}=T_{1}(4) T_{5}(3) \cdots T_{3 l+2}(3) I_{3 l+5}[3(k-l-1)] \\
& \sum C_{j}=\left(\frac{9}{2} k^{2}-9 k l-\frac{15}{2} k+\frac{15}{2} l^{2}+\frac{35}{2} l+12\right) P_{1} \\
& +\left(9 k l-\frac{15}{2} l^{2}-\frac{37}{2} l+15 k-11\right) P_{2}
\end{aligned}
$$

Proof

$$
\begin{aligned}
& \sum_{j \leq 3 l+4} C_{j}=\frac{1}{2}[3(l+1)+5](l+1) P_{2}+\left[3(l+1)^{2}+4(l+1)+2\right] P_{2} \\
& \sum_{j>3 l+4} C_{j}=\sum_{u=3 l+5}^{3 k+1}\left\{u P_{1}+\left[(3 l+5) P_{2}-(3 l+4) P_{1}\right]\right\} \quad \text { Then }
\end{aligned}
$$$$
\text { ; }
$$$$
=3(k-l-1)\left[\frac{3}{2}(k-l) P_{1}-P_{1}+3 l P_{2}+5 P_{2}\right]
$$$$
\sum C_{j}=\sum_{j \leq 3 l+4} C_{j}+\sum_{j>3 l+4} C_{j} .
$$

Theorem 4 Let $v=\frac{k}{5}+\frac{2 P_{2}}{15\left(P_{1}-P_{2}\right)}-\frac{4}{3}$, when $l$

satisfy $v<l<k-1$,

the total flow-time of the schedule

$S_{2}=T_{1}(4) T_{5}(3) \cdots T_{3 l+2}(3) I_{3 l+5}[3(k-l-1)]$ is lesser than the schedule $S_{*}=T_{1}(4) T_{5}(3) \cdots T_{3 k-1}(3)$.

Proof From lemma1 and lemma2, the difference between the total flow-time of the schedule $S_{*}$ and $S_{2}$ is

$$
\sum_{S_{k}} C_{j}-\sum_{S_{2}} C_{j}=\left[\left(P_{2}-P_{1}\right)\left(\frac{3}{2} k-\frac{15}{2} l-10\right)-P_{2}\right](k-l-1)
$$

As

result,

when

$v=\frac{k}{5}+\frac{2 P_{2}}{15\left(P_{1}-P_{2}\right)}-\frac{4}{3}<l<k-1, \sum_{S_{k}} C_{j}-\sum_{S_{2}} C_{j}>0$, that is $\sum_{S_{*}} C_{j}>\sum_{S_{2}} C_{j}$.

Finally, we consider $n=3 k$.After completion of the processing job $3 l$, processing job $3 l+1$ in $M_{2}$ in first thing, after a period of idle time $t=(3 l+1) P_{2}-3 l P_{1}$ in $M_{1}$, processing job $3 l+1,3 l+2, \cdots, 3 k+2$ in $M_{1}$ continuously, processing job $3 l+2,3 l+3, \cdots, 3 k+2$ in $M_{2} \quad$ continuously. It is that turn schedule $\quad S_{*}=T(3) T(3) \cdots T(3) Q(5) \quad$ to $S_{3}=T_{1}(3) T_{4}(3) \cdots T_{3 l-2}(3) I_{3 l+1}[(3 k+2)-3 l]$, among $S_{3}$ there is idle time $t=(3 l+1) P_{2}-3 l P_{1}$ in $M_{1}$ after completion of the processing job $3 l$.

Lemma 4 The total flow-time of the schedule

$$
S_{3}=T_{1}(3) T_{4}(3) \cdots T_{3 l-2}(3) I_{3 l+1}[(3 k+2)-3 l]
$$

is $\sum C_{j}=\left(\frac{15}{2} l^{2}+\frac{9}{2} k^{2}-9 k l+\frac{15}{2} k-\frac{11}{2} l+3\right) P_{1}$

$$
+\left(9 k l-\frac{15}{2} l^{2}+\frac{9}{2} l+3 k+2\right) P_{2}
$$

Proof: $\sum_{j \leq 3 l} C_{j}=\frac{3}{2} l(l+1) P_{2}+l(3 l+2) P_{1}$,

$\sum_{j>3 l} C_{j}=\sum_{u=3 l+1}^{3 k+2}\left[u P_{1}+(3 l+1) P_{2}-3 l P_{1}\right]$

$=(3 k-3 l+2)\left[\frac{3}{2}(k-l+1) P_{1}+(3 l+1) P_{2}\right]$,

then $\sum C_{j}=\sum_{j \leq 3 l} C_{j}+\sum_{j>3 l} C_{j}$.

Theorem 5 Let $l<k$,when $l$ satisfy

$\left(\frac{3}{2} k^{2}-9 k l+\frac{15}{2} l^{2}\right)\left(P_{2}-P_{1}\right)+\left(\frac{11}{2} l-\frac{5}{2} k\right) P_{1}+\left(\frac{3}{2} k-\frac{9}{2} l\right) P_{2}>0$

The total flow-time of the schedule $S_{3}=T_{1}(3) T_{4}(3) \cdots T_{3 l-2}(3) I_{3 l+1}[(3 k+2)-3 l]$ is lesser than the schedule $S_{*}=T(3) T(3) \cdots T(3) Q(5)$.

Proof:From lemma 1 and lemma 4, the difference between the total flow-time of the schedule $S_{*}$ and $S_{2}$ is

$\sum_{S_{*}} C_{j}-\sum_{S_{3}} C_{j}=\left[\left(\frac{3}{2} k^{2}-9 k l+\frac{15}{2} l^{2}\right)\left(P_{2}-P_{1}\right)+\left(\frac{11}{2} l-\frac{5}{2} k\right) P_{1}+\left(\frac{3}{2} k-\frac{9}{2} l\right) P_{2}\right.$

There is the conclusion of theorem 5 .

\section{EXAMPLES}

Example 1

$n=18, P_{1}=22, P_{2}=21, k=\frac{n}{3}=6, v=\frac{6}{5}+\frac{41}{15}=\frac{59}{15}$.

The total flow-time of the schedule

$S_{*}=T_{1}(3) T_{4}(3) T_{7}(3) T_{10}(3) T_{13}(3) T_{16}(3)$ is $\sum C_{j}=3963$. Let $l=4$, the total flow-time of the schedule

$$
S_{1}^{\prime}=T_{1}(3) T_{4}(3) T_{7}(3) T_{10}(3) I_{13}(6) \quad \text { is } \quad \sum C_{j}=3962,
$$

among $S_{1}^{\prime}$ there is idle time 9 units in $M_{1}$ after completion of the processing job 12. Let $l=5$, the total flow-time of the schedule $S_{1}^{\prime \prime}=T_{1}(3) T_{4}(3) T_{7}(3) T_{10}(3) T_{13}(3) I_{16}(3) \quad$ is $\sum C_{j}=3955$, among $S_{1}^{\prime \prime}$ there is idle time 6 units in $M_{1}$ after completion of the processing job 15 .

Example 2

$$
n=19, P_{1}=22, P_{2}=21, k=\frac{n-1}{3}=6, v=\frac{8}{3} .
$$

The total flow-time of the schedule $S_{*}=T_{1}(4) T_{5}(3) T_{8}(3) T_{11}(3) T_{14}(3) T_{17}(3)$ is 
$\sum C_{j}=4397$.Let $l=3$, the total flow-time of the schedule

$$
S_{2}^{\prime}=T_{1}(4) T_{5}(3) T_{8}(3) T_{11}(3) I_{14}(6) \quad \text { is } \quad \sum C_{j}=4392
$$

among $S_{2}^{\prime}$ there is idle time 8 units in $M_{1}$ after completion of the processing job 13. Let $l=4$, the total flow-time of the schedule

$$
S_{2}^{\prime \prime}=T_{1}(4) T_{5}(3) T_{8}(3) T_{11}(3) T_{14}(3) I_{17}(3)
$$

$\sum C_{j}=4387$, among $S_{2}^{\prime \prime}$ there is idle time 5 units in $M_{1}$ after completion of the processing job 15 .

\section{REFERENCES}

[1] Lawer,E.L.et al,Sequencing and Scheduling: A lgorithms and Complexity, S .G.Graves et al,Eds.,Handbooks in OR \& MS, Vol4,1993,445-522.

[2] J.O.Achugbue,F.Y.Chin,Scheduling the open-shop to minimize mean flow time. SIAM J.Comput.11,1982,709-720.

[3] M.Dror, Open shop scheduling with machine depedent processing times,Discrete Applied Mathematics,39,1992,197-205.

[4] Siming Xiang,Guochun Tang,Open-Shop Scheduling Problem with Machine Depedent Processing Times ,OR Transactions, 1998,2(1),71-78..

[5] Wenci Yu,Gang Ying,On the Total Flow-Time Problem of Two Machine Open-Shop ,OR Transactions, 1998,2(2),84-94 